\title{
Child nutrition and growth: butterfly effects?
}

\author{
France Bellisle* \\ INSERM U557/INRA U1125/CNAM EA3200/Univ Paris 13, Centre de Recherche en Nutrition Humaine d'Ile-de France, Unité de \\ Recherche en Epidémiologie Nutritionnelle, SMBH, 74 rue Marcel Cachin, Bobigny cedex 93017, France
}

The international symposium organised by the Danone Institute as a pre-congress satellite of the European Nutrition Societies Congress, in Paris, in July 2007, brought together experts of child nutrition and behaviour. These experts coming from several countries of Europe and North America shared their views on the impact of numerous factors affecting child eating and health in present-day developed societies. Topics included nutritional influences during foetal life, early life development of food likes and dislikes, neophobia, ability for energy regulation, impact of media and advertisement, etc. Most contributions addressed the obesity epidemics and the problems associated with body weight control. Other aspects of child health were also considered, for example the nutritional challenge of adequately feeding children with type 1 diabetes. The nine speakers were asked to underline practical strategies to improve nutrition in early life, so as to maximise health, growth and quality of life in today's environment. The present paper is a summary of the ideas expressed by the experts, highlighting agreements, convergences, and occasional disagreements between expert opinions.

\section{Nutrition: Child: Growth: Body weight control}

The recent symposium organised by the Danone Institute International as a satellite to the FENS 2007 Congress brought together distinguished scientists from many areas of the world with expertise in several areas of research and care related to child growth and health. A central theme to most of the talks was body weight control in growing children although other health issues were considered. The talks highlighted the crucial importance of nutritional factors following and before birth. They revealed how the foetus, the infant, and the child are exposed to countless influences from foods ingested by the pregnant mother or by the young person at various stages of development. Our speakers all agreed on the paramount importance of nutritional factors for child development and weight control but clearly had differing, if not diverging opinions about the relative contribution of critical factors: the mother, the family, the society, the environment and particularly the school environment, genetics, economics, and others. Prevention and treatment of overweight also elicited varied opinions.

Curiously, in spite of the very different geographic origins of our speakers, not much clash occurred that could be attributed to different influences in various parts of the developed world (Europe and North America). It is as if our children, who benefit the best conditions in history to enjoy a harmonious and healthy growth, were under the influence of a host of more or less visible sources of trouble that negatively affected their health, inducing unprecedented frequencies of nutrition-related conditions such as overweight and diabetes. Are we dealing with a special case of the "butterfly effect" that has been demonstrated under different situations: even the smallest effects such as the seemingly random deviations in the flight of a butterfly in one part of the world can trigger major weather catastrophes such as tornadoes or tsunamis in other parts of the world. Some of our speakers actually did mention the butterfly effects and their collective contribution to our symposium suggests that we might be dealing with a number of wandering butterflies whose flights interact in perhaps inextricable manners. It does seem obvious that the effects of such interactions do create catastrophes in terms of child health and development worldwide. Our speakers, after describing the particular kinds of individual or social factors they were experts in, were invited to suggest a few ideas of practical solutions that could be used to understand the impact of butterfly wanderings in their fields and potentially correct their deleterious effects.

This was done, and at the end of our one-day symposium we had a large number of ideas that did temper the sensation of helplessness commonly found in the scientific and lay literature dealing with the present epidemic of overweight and other nutrition-related problems in young people all over the world. This final chapter of the proceedings of the symposium will review these suggestions and try to identify how they could be used, in isolation or in combination, to prevent nutrition-related problems in early life and perhaps even correct them before the child develops into an adult with poor quality of life and faces the numerous long-term complications originating from early life conditions.

\section{Genetics, foetal and neonatal influences}

Our first speaker, Dr Luc Marlier, presented work on newborns showing that prenatal sensory influences had an 
impact on orientation responses to olfactory stimulation. Taste buds as well as olfactory receptors are functional in the third semester of pregnancy. The foetus therefore gets exposed to a variety of taste and smell stimuli that depend on the mother's diet. Prenatal exposure affects postnatal responses to a variety of olfactory stimuli. In Dr Marlier's words, culture is present in the amniotic fluid. It has been clearly showed that human newborns orient to the odour of the amniotic fluid and the colostrum. A few hours after birth, infants of mothers who ingested anis-smelling substances during pregnancy, orient to anis-smelling stimuli. In contrast, infants of women who did not ingest anis-smelling substances reject anis stimuli four days after birth. Garlic and alcohol intake by pregnant women could likewise affect the newborn's responses. Even pre-term infants respond to olfactory stimuli such as vanilla by increasing their respiratory rate. These fascinating observations clearly demonstrate that sensory responses can be affected by prenatal influences originating from the mother's food choices; however they do not tell us whether such responses can affect food preferences, food choices, or food intake in the offspring. Clearly, more research is needed in this field. The data are nevertheless extremely precious and converge with other recent evidence highlighting the importance of early life events.

The notion that genetic factors were crucial in every aspects of a person's life is well-known if not universally accepted. The nature-nurture argument has raged for decades and is not solved today. It has even gained in complexity in recent years as the new developments in genetic research have allowed an improved appreciation of the impact of genes on particular traits. We now know that very few individual characteristics depend on a single gene and that most are affected by a variety of genes. In addition, the importance of "epigenetic" factors has been demonstrated. Genes are expressed in certain environments and remain unexpressed in others. The critical factors of the environment that permit or inhibit the expression of genes also remain to be identified in many aspects of growth and health. This is why much research is devoted today to early life factors, among which dietary conditions. Nutritional influences during pregnancy could be some of the critical factors allowing the expression of certain genes. More ambitious hypotheses even include the nutritional status of the mother-to-be before she becomes pregnant. Such factors affect a number of events occurring in early life, some of which are becoming increasingly recognised as determining the future health and development of the child. Birth weight is the subject of much very stimulating research as it has become evident that both low and high birth weight can predict future weight control problems and other diseases.

We already see a multitude of butterflies interacting over the unborn infant to influence future life events. Genes, mother's dietary and health status, epigenetic factors, among others, set the scene for the infant's first moments of life and already determine important aspects of the young person's chances to enjoy a healthy development. Although it remains difficult to counteract the influence of genes, awaiting future understanding of genetic and epigenetic effects, it seems possible to act on young women's nutritional status before and during pregnancy. Public health authorities and professionals know how difficult it is to make young persons, including future mothers, adopt a healthy lifestyle. Individual and social factors can either help or create problems. Some of such factors were addressed by our other speakers.

\section{The impact of society: cognitive schemas}

Beyond infancy, children have to learn to survive in a given society. In rich developed societies, recent decades have seen marked changes in many aspects of lifestyle. While energy needs are decreasing due to increasingly sedentary work and leisure activities, the food supply has increased in amount, variety and convenience. For reasons that have to do with both sensory appeal and nutritional input, foods that are rich in energy, high in fat and/or sugar, are easily accepted and ingested by children. It is clearly more difficult to have children accept foods generally regarded as "healthier", such as vegetables, fruits, fish, etc. This is a clear problem for parent, educators, and health professionals. Where can we find hope? How can we make our children more willing to try and perhaps enjoy such healthy foods, rather than overindulging in high energy substances that can create weight control or health problems in those with the wrong genetic background or unfavourable life circumstances? In a society, children and adults have particular cognitive attitudes or schemas about substances that can be ingested as foods. Such schemas can influence behaviours to a large degree and were the topic of Dr Patricia Pliner's talk. Dr Pliner defined such schemas as mental structures that represent organised knowledge about a given type of stimulus, and in particular foods. They affect expectations about familiar and unfamiliar foods. Knowing how cognitive schemas work can help us improve our children's acceptance of diverse and unfamiliar foods.

One type of behaviour found in numerous children in developed countries, in Europe as well as in America, is called "neophobia" and is characterised by a refusal expressed by the child to accept objects, among which foods, that are unfamiliar or novel. Most (but not all) children, between the ages of 2 and 10 years, are affected by more or less severe neophobia. Some cultures might be more permissive and expose young children to a broader variety of food stimuli, thereby making more foods seem familiar to the child. Studies should be carried out on this issue and it would be interesting to compare how easy (or how difficult) it is in different countries of the developed world to induce acceptance or even liking for a broad variety of foods including those that have a low appeal to many children. In all countries, nevertheless, some proportion of children do exhibit neophobia and strongly reject many foods that their parents regard as "healthy".

Children use "cognitive schemas" to evaluate foods. When a novel or unfamiliar food is presented, many children have the cognitive schema that it will not taste good. Convincing the child that the schema does not apply to this novel food and that this food is an "exception" to the general schema could facilitate the child's willingness to taste the food and have a chance to realise that its taste is not indeed unpleasant. Another strategy could be to convince the child that the food actually does not belong in the novel, unknown category, but rather is a familiar food. The trick to achieve such a cognitive change in the child is to use what Paul Rozin has called a locally familiar "flavour principle"(1). In every culture, certain 
foods, spices, or other salient flavour substances are used in many staple foods. Every child learns early in life that foods with such flavour characteristics are both tasty and safe. Examples are chilli in Mexico, tomato and olive oil in Mediterranean countries, curry in India. Children who have observed adults and peers enjoy foods with those culturespecific flavour principles are willing to ingest them and, following experience of the taste and post-ingestive consequences of intake, readily develop strong preferences for foods that are typical of their own cultures.

Observations of migrant populations have revealed that food traditions survive longer after people have moved to a different country than other types of original cultural traditions. A novel food that is presented under the "disguise" of an accepted flavour principle is more likely to be accepted and perhaps liked than a food that is experienced as a totally unknown object whose sensory characteristics might be unpleasant. Role models are also important: witnessing significant others enjoy the food can encourage the child to test the food. The mother is the best model.

A more challenging strategy would be to change the child's cognitive schemas about novel foods and create expectations that novel foods can indeed be good-tasting, appealing and safe. Although research is rare about the mechanisms that could induce such a change, it has been shown that exposing the child to good-tasting novel foods does increase the willingness to try novel foods. Early exposure to a broad variety of foods may make the child less neophobic: variety of food choices made available to a young child can create cognitive schemas that novel foods actually taste good.

Clearly, more research is needed about neophobia in children and its cultural, geographic differences. What is known however is that neophobia usually decreases as the child grows up and has disappeared in most by age 12 years. Some children actually exhibit little or no neophobia but the reasons that account for the specific intensity of the symptom in different individuals remain to be elucidated. Genetic, epigenetic, socio-cultural or family factors could interact and exert still unknown levels of influence.

\section{The special problem of fruit and vegetable intake}

The specific case of fruits and vegetable intake was addressed by Dr Hans Brug. This is obviously a case of clear disagreement between recommendations and behaviours. Only about $17.6 \%$ of eleven year-olds in Europe do comply with the recommended daily intake of five fruits or vegetables a day. Dr Brug analysed the reasons for the discrepancy and the strategies that could help bridge the important gap between recommended and actual intake. Three crucial determinants that could be modifiable to a certain extent are motivation, ability and availability. The motivation to ingest vegetables is usually low in young people because their sensory characteristics are not those that appeal to children (sweetness, fatness, softness, etc.) and the same is true to a lesser degree of many fruits.

Children's responses to the taste of fruits and vegetables could be improved via a number of strategies of demonstrated efficiency: mere exposure, pairing the vegetable with other foods of high palatability (sensory-sensory learning), social modelling could be used. The ability of the parents and/or caregivers to propose, prepare, cook vegetables in a more pleasant way could be improved and the importance of doing so could be highlighted by public information campaigns. Environmental opportunities should also be improved. One problem with vegetables is their price and the time needed to cook them. Dr Brug recommends intervention programs involving both school-based and family activities. The ultimate goal is to teach children to like fruits and vegetables, which requires that children are exposed to them in situations that are experienced as pleasant, safe, and free of conflict. This obviously involves large social and economic changes, so that ability and availability will in fact modify children's attitudes and motivations to these foods.

Dr Wardle extended the argument to underline that the problem is probably different for fruits and vegetables, since most children like fruits (at least some of them) while they dislike vegetables. This probably has to do more with sensory factors (sweetness, texture, etc.) than with availability or price. The innate acceptance of sweetness and rejection of bitterness can orient children's food choices for many years in early life. Another potentially critical factor is the energy density of foods. According to a physiological reinforcement theory, energy rich foods, or in other words foods with a high energy density, are easily accepted by children because such foods provide the high input of energy that growing children need. Given the high frequency of child overweight, it seems that our children do get plenty of energy to meet their high needs and many actually get too much. In such a case, the same physiological homeostatic theory could predict that once the energy needs are met, then children should turn away from high energy density foods and appreciate foods with lower energy content but higher nutrient density. Obviously this is not what happens. Even the notion of "sensory specific satiety" would predict that children would turn to different types of foods after ingesting plenty of high sugar, high fat foods, but this again does not happen, perhaps due to the immense variety of available energy rich sweet or savoury foods.

Under such a situation, Dr Wardle proposed her strategies for facilitating acceptance of fruits and vegetables by children. She believes in family influence and in the parent-child or child-sibling relationships that can provide useful role models. In these efforts, with the same level of exposure, some children are less responsive than others and exhibit high levels of neophobia (fussiness). Dr Wardle exposed evidence that the intensity of neophobia might be determined by genetic factors. Studies of same sex twin pairs showed that monozygotic twins aged 4-5y had higher correlations of their food likes than dizygotic twins. A study of 5406 twin pairs revealed that the score on a neophobia scale at age $10 \mathrm{y}$ was higher in mono than dizygotic twins. The neophobia score is a strong predictor of acceptance of vegetables in children. The heritability of neophobia could be as high as 0.78 . If neophobia and rejection of vegetables is determined by genetic factors, then what can be done?

Understanding the causes of such a rejection response is a first step. Clearly, sensory factors are important and fussier children could happen to be more sensitive to the bitter or sour taste of some vegetables or other foods. Some children may be more prone to digestive disorders than others and the association of salient sensory cues from unfamiliar foods could become associated to unpleasant post-ingestive 
disorders, such as nausea. Clearly we need more information about what makes some children fussier than others and more research is needed. What seems obvious, given the level of acquired knowledge, is that fruits and vegetables should be available often, both on the family table and at school meals. Making them palatable is important, even though this may require time and culinary skills. They could also be offered as snacks in a pleasant way. Social facilitation by witnessing significant role models ingest the foods may be an efficient strategy to induce long-term progresses in acceptance. They should be prepared and presented in a way that will make them acceptable to children, and the high sensitivity of some children to bitter, for example, should not be antagonized by threats or constraints.

\section{Food choices and energy regulation}

The foods children enjoy and accept are a very important aspect of their ability to obtain a healthy nutritional status. Another problem, which was briefly alluded to above, is the amount of food children are willing to eat. There is supportive scientific evidence that children are rather apt at adjusting their energy intake to their energy needs. Pioneer works by Leann Birch $^{(2)}$ and others, have established that young children are able to adjust their energy intake according to what they have ingested in the previous hours. The "pre-load paradigm" has shown that after ingesting a high energy pre-load, pre-schoolers spontaneously eat less than after the intake of a low energy pre-load. It is also generally held that adults and elderly persons are less able to achieve such a corrective adaptation of their intake, although the very few studies that have looked at this problem usually report large inter-individual variations in the ability to adequately compensate for the energy ingested previously. It also seems that it is easier to compensate for a low energy preload (by eating more at the next occasion) than for an excess of energy (by eating less at the next occasion).

Today, the question of the ability of humans to adequately compensate for energy variations in intake is still a debated issue. Most data indicate that compensation or adjustment is approximate at best, and can be very poor in many people. Infants and children may be better than adults, but they progressively lose this precious ability to respond to their internal cues signalling energy needs as they grow and learn to eat according to externally determined social rules. It would be very useful to maintain and even improve the energy compensation abilities in most people. Dr Susan Johnson reviewed the literature dealing with this extremely important topic and highlighted that several factors can affect results of energy adjustment studies: age, gender, previous learning, environmental factors and experimental design, among others. Research over the years confirms the large inter-individual differences even in young children and the age-related decline in the ability to adjust energy intake to needs. When no agerelated difference is reported, it is because adjustment is just very poor in everybody ${ }^{(3)}$. When there is compensation, it is not perfect and generally biased toward over rather than undereating. The constant availability of palatable, convenient, low cost, energy rich foods may counteract any compensation ability a child could exhibit under less permissive circumstances.
Dr Johnson underlined the very important influence of the number of occasions to eat on total energy intake: children eat in response to opportunity ${ }^{(4)}$. How much is served (portion size) and how often food is available could be two important factors affecting the ability of a person, including a child, to effectively adjust energy intake as a function of energy needs. Supportive evidence highlights the strong impact of environmental influences, regardless of innate or genetically determined characteristics: portion size, meal frequency and duration, exposure to food cues in the environment and particularly in the media, etc. More research is needed on the effects of these factors, both in isolation and in interaction.

\section{Clinical practice}

Dr Silvia Scaglioni is a clinician dealing with obese children. She offered some practical advice that could be useful to both parents and health professionals. Dr Scaglioni underlined the crucial role of parents in providing healthy foods but recognised the increasing difficulty of this task in our obesogenic environment in which inexpensive high energy density food is constantly available. Among the behaviours that can induce problems is the too frequent offering of foods to children in response to stress or as a reward. Dr Scaglioni advocates judicious parental modelling of good eating practices, both in terms of food choices and eating frequency. In her view, both parents of an overweight child should participate in the efforts to improve the child's behaviour. They should be made aware of the long term consequences of child obesity. While information is important, it is not sufficient to modify behaviours. Therefore long term programmes of behavioural changes are to be developed, integrating the child's own suggestions.

One strategy is to ask the overweight child what he/she would advise another overweight kid to do in order to lose weight. Principles of sound behavioural modification techniques should be applied: small steps at a time, small obtainable goals, progressively more ambitious changes and goals, constant and positive support from the family, etc. Dr Scaglioni suggested that instructing mothers before delivery about child growth and nutrition would be useful. It would be particularly important to help overweight mothersto-be and women with eating behaviour problems to solve these problems as much as possible before the child is born. This obviously requests a strong level of support and organisation not only from health professionals but from the society as a whole.

\section{Education in practice}

Dr Jana Parizkova is also a great believer in education of parents as well as children. She presented data from her country, the Czech Republic, underlining something that is also well known in other countries: well educated people are usually leaner and healthier than people with low education levels. Mothers' education level has impact on the foetus and later on, on the infant during the first few months of life. Parents' education level is linked with breastfeeding and with the amount of physical activity that the child is encouraged to engage in, as confirmed by a recent study of about 10000 Czech children. A key factor in addressing the 
obesity epidemics, according to Dr Parizkova, is to educate the mothers.

We are grateful to Dr Parizkova to have reminded us that our present cogitations can be traced back to the thinking of the great Czech pedagogue Comenius who, in 1632, already proposed the basic advice we are still desperately trying to convince our contemporaries to adopt: the body energy balance depends on both healthy adequate eating and sufficient physical activity. Comenius's wise advice has been supported, rather than weakened, by later research but over the last 400 years we have not succeeded in creating a society in which behaviours allowing an adequate energy balance could be willingly practiced by all, adults and children.

Dr Parizkova presented data showing that spas for obese children could be an excellent place to teach them adequate behaviours, including food choices, eating frequency, and physical exercise. Interesting medium-term results have recently been reported by other teams ${ }^{(5)}$ confirming Dr Parizkova's observations. Such studies show that, under appropriate circumstances, children and adolescents can in fact be taught to behave in a weight-control friendly way. The problem is that the society they return to after they have lost considerable amounts of weight does not support the healthy behaviours that they have learned to perform under a totally different environment. Here again, parental support is of paramount importance.

\section{Pathologies in normal weight children: the example of type} 1 diabetes mellitus

Dr Claudio Maffeis is a paediatrician who addressed the many problems associated with type 1 diabetes mellitus in children. This severe disease, with heavy long-term threats on health and quality of life, is more and more frequent in children and appears at an increasingly earlier age. Although it is not directly caused by overeating, as is type 2 diabetes mellitus found increasingly often in young individuals, the problem of adequate nutrition is an extremely important aspect of treatment of type 1 diabetes mellitus in children and adolescents. Type 1 diabetes mellitus has a known genetic basis which is facilitated by environmental triggers, among which inappropriate nutritional conditions. It is generally held that encouraging an optimal nutrition in children, both in terms of food choices and energy intake, can reduce the rates of heavy complications in later life. Nutrition exerts its beneficial (or detrimental) effects by affecting glucose control. Public health authorities in developed countries, such as the American Diabetes Association, advocate nutrition therapy as a crucial part of treatment. The nutritional needs of growing children should be covered both in terms of quantity and quality. Dr Maffeis underlined that the severe health consequences of type 1 diabetes: inflammation and lipotoxicity contributing to the development of the metabolic syndrome, micro and macroangiopathy, etc. Such complications can get extremely difficult to cope with if they are allowed to develop from early life.

In order to maintain the pleasure of eating, which is essential for obtaining long-term compliance to the demanding diet, an individualised approach to dietary advice is recommended, as already suggested by Dr Scaglioni. Dr Maffeis advocates a complex treatment that includes both dietary aspects and physical activity. Obviously, carbohydrate intake has to be monitored, as patients with type 1 diabetes cannot secrete insulin and cannot benefit from physiological glycemic control. The large amount of knowledge recently acquired about the glycemic index and the glycemic load should allow parents to facilitate food choices in order to optimize the child's glycemic levels ${ }^{(6)}$. Again, the important contribution from family members was stressed, in particular the interactions between family members at mealtimes.

One of the main problems of treating children and adolescents with type 1 diabetes is the commonly poor adherence to several aspects of treatment, such as insulin injections and dietary advice. While children are extremely sensitive to taste factors, as already underlined by previous speakers, adolescents also want and need to join groups of peers. Given the generally poor eating practices of this age group, adolescents with type 1 diabetes might be exposed to very bad influences that must be acknowledged, understood and neutralised as much as possible. This is an extremely difficult medical problem with potential critical consequences for the patients and for society as a whole. More research is clearly needed on the conditions of a better compliance with dietary recommendations in early life as this can affect many other future health consequences than body weight control.

\section{Young people and food advertising}

Our last speaker addressed a topic that is not frequently examined in scientific conferences: that of advertising and market research dealing with children consumers. Ms Immaculata Garcia Alvarez confirmed that young people are exposed to much encouragement to consume high energy density foods by the media, particularly television. Most television commercials targeted at children are about foods $(56 \%)$ and $46 \%$ are about high fat, high sugar items. Only $4 \%$ deal with foods that could be considered part of the traditional "Mediterranean diet". The impact of such advertisements on food choices and behaviours remains uncertain however, as no correlation was found between the percentage of food advertisement and the overweight rate in children populations. Ms Garcia Alvarez believes that the strategies used to affect food choices in advertisement could be used to encourage the intake of "healthy" foods, using the same message contents: having fun, ideal families, associating certain foods with characters or individuals that children admire, etc. Given the very high costs of advertising in the media, it appears difficult to see how governments or public health authorities could compete with the food industry to encourage healthy food choices.

As also underlined by Ms Garcia Alvarez, recent societal changes have facilitated inadequate changes in the way children are fed: both parents work long hours, they have little time and energy to prepare "Mediterranean" type family meals, they experience guilt over this, and often show excessive permissiveness for questionable behaviours as nibbling. One problem comes from the fact that traditional cooking practices are no longer transmitted over generations, and that many places where children are fed such as school cafeterias propose unpalatable foods. This has made convenient inexpensive snack foods even more attractive.

Some experts openly speak of a toxic environment. It is unlikely that recent social changes could or should be reversed. 
The way forward is in the direction of more social changes that would require a convergent involvement of all significant actors: parents, educators, public health authorities, industry, media, etc. The relative importance of every party's responsibility is the subject of fierce argument among experts and the lay public. Scapegoats are frequently designated: the food industry, school cafeterias, the media, etc. Such discussion could go on forever, while our children are getting fatter and more vulnerable to nutrition-related diseases. While many of our speakers underlined the crucial importance of the family and especially the mother, it remains clear that motherly influence occurs in a given society that also exerts strong impact on individual behaviours. Societal factors may impose very difficult conditions to individual goodwill; conversely, some of the individual's responsibility has to do with creating the societal conditions in which children grow up.

\section{Conclusions: a few practical considerations}

Our symposium lasted one very full day. Many aspects of child nutrition were addressed but, obviously, many were not even alluded to. For example, the nutrient imbalance experienced by infants at weaning, when the high-fat mother's milk is suddenly replaced by a very high protein $\operatorname{diet}^{(7)}$, has not been discussed. The influence of low or high birth weight is another determining factor of many future health and weight control consequences. So, acknowledging that many important aspects were not included in our analysis of children's feeding conditions in developed societies, a few practical ideas were expressed and discussed by our speakers.

While genetic factors are clearly important, epigenetic influences should be studied and optimised. This involves providing high quality information to the public and in particular to women of child-bearing age. Pre-pregnancy nutritional status of mothers-to-be deserves consideration. Events occurring during pregnancy are of unquestioned importance. Early life nutritional events can influence food choices and eating behaviours later on. Exposing children to a variety of healthy palatable foods under convivial circumstances could create good habits. While everyone agreed that the mother, and more broadly the family, had a crucial impact, the role of societal factors was also recognised. The influence of peers, school, the media and the food industry was recognised by all, although the relative contribution of each could be discussed. Society as a whole has a central role in making the conditions of availability of healthy food choices and opportunities for physical exercise accessible to all, including to families with low income and limited capacity to understand complex nutritional information.

\section{Conflict of interest statement}

Dr Bellisle received honoraria from the Institute as an organiser of the symposium, as a speaker and as an invited editor for the special issue of the journal. The author has no conflicts of interest to report.

\section{References}

1. Stallberg-White $C \&$ Pliner $P$ (1999) The effect of flavor principles on willingness to taste novel foods. Appetite 33, 209-221.

2. Birch LL \& Fisher JO (1998) Development of eating behaviors among children and adolescents. Pediatrics 101, 539-549.

3. Zandstra EH, Mathey MF, de Graaf C \& van Stavaren WA (2004) Short-term regulation of food intake in children, young adults and the elderly. Eur J Clin Nutr 54, 239-246.

4. Mrdjenovic G \& Levitsly DA (2005) Children eat what they are served: the imprecise regulation of energy intake. Appetite 44, 273-282.

5. Rolland-Cachera MF, Thibault H, Souberbielle JC, Soulié D, Carbonel P, Deheeger M, Roinsol D, Longueville E, Bellisle F \& Serog P (2004) Massive obesity in adolescents: dietary interventions and behaviours associated with weight regain at $2 \mathrm{y}$ follow-up. Int J Obes Relat Metab Disord 28, 514-519.

6. Wolever TM \& Brand-Miller JC (2005) Influence of glycemic index/load on glycemic response, appetite, and food intake in healthy humans. Diabetes Care 28, 2123-2129.

7. Rolland-Cachera MF, Deheeger M, Maillot M \& Bellisle F (2006) Early obesity rebound: causes and consequences for obesity in children and adults. Int J Obes 30, Suppl. 4, S11-S17. 